\title{
Macrophages and endothelial cells orchestrate tumor-associated angiogenesis in oral cancer via hedgehog pathway activation
}

\author{
Ludmila de Faro Valverde ${ }^{1}$ • Thiago de Almeida Pereira ${ }^{2} \cdot$ Rosane Borges Dias $^{1}$. \\ Vanessa Sousa Nazaré Guimarães ${ }^{1}$. Eduardo Antônio Gonçalves Ramos ${ }^{1}$ • \\ Jean Nunes $\operatorname{Santos}^{3}$ - Clarissa Araújo Gurgel Rocha ${ }^{1,3}$
}

\begin{abstract}
The present study aimed to evaluate the role of Hedgehog $(\mathrm{Hh})$ molecule expression in association with the clinical aspects of oral squamous cell carcinoma (OSCC), as well as angiogenesis and CD163+ macrophages. Twentyeight cases of OSCC, nine cases of tumor-free resection margins (TM), and four cases of non-neoplastic oral mucosa (NNM) were submitted to immunohistochemistry to detect proteins Sonic Hedgehog (SHH), Indian Hedgehog (IHH), GLI1, CD163, and CD105. Protein colocalization with respect to SHH/CD163, IHH/CD163, GLI1/CD163, and GLI1/CD105 was assessed by immunohistochemical double staining. In tumor parenchyma, SHH and IHH were present in the cytoplasm of neoplastic cells, while GLI1 was observed in cytoplasm and nucleus. Endothelial cells were found to express $\mathrm{SHH}, \mathrm{IHH}$, and GLI1 within CD105+ vessels, and a positive correlation between infiltrating macrophage density (IMD) and microvascular density (MVD) was observed in cases of OSCC and TM. When compared to TM and NNM,
\end{abstract}

Electronic supplementary material The online version of this article (doi:10.1007/s13277-015-4763-6) contains supplementary material, which is available to authorized users.

Clarissa Araújo Gurgel Rocha

gurgel.clarissa@gmail.com; cgurgel@bahia.fiocruz.br

1 Laboratory of Pathology and Molecular Biology, Oswaldo Cruz Foundation, Gonçalo Moniz Research Center, R. Waldemar Falcão, 121, Candeal, Salvador, Bahia CEP: 40296-710, Brazil

2 Laboratory of Experimental Pathology, Oswaldo Cruz Foundation, Salvador, Bahia, Brazil

3 Laboratory of Oral Surgical Pathology, School of Dentistry of the Federal University of Bahia, Salvador, Bahia, Brazil the OSCC cases demonstrated higher immunoreactivity for SHH $(p=0.01)$, IHH $(p=0.39)$, GLI1 $(p=0.03)$, IMD $(p=0.0002)$, and MVD $(p=0.0002)$. Our results suggest the participation of the Hh pathway in OSCC by way of autocrine and paracrine signaling, in addition to the participation of both $\mathrm{SHH}$ and IHH ligands. Endothelial cells were also found to exhibit positivity with respect to Hh pathway components and we surmise that these molecules may play a role in tumor angiogenesis. CD163+ macrophages were also observed to express $\mathrm{IHH}$, a ligand of this pathway, in addition to being associated with tumor neovascularization.

Keywords Oral cancer · Squamous cell carcinoma · Hedgehog proteins $\cdot$ Immunohistochemistry

\section{Introduction}

Oral cancer is among the ten most common forms of cancer occurring worldwide. The predominant histological type is oral squamous cell carcinoma (OSCC), which corresponds to over $95 \%$ of all diagnosed cases [1,2]. At advanced stages, this disease is associated with elevated morbidity and mortality, as fewer than $50 \%$ of patients survive longer than 5 years $[3,4]$.

In light of the conceptual evolution surrounding the development of malignant neoplasia, particularly with respect to cancer stem cells, the study of embryonic signaling pathways holds great promise, notably as a strategy to identify relevant biomarkers. In this context, dysregulation of the Hedgehog (Hh) embryonic signaling pathway has been associated with the development and progression of a range of human tumors [5-8]. Additionally, the abnormal activation of this signaling cascade has previously been described in OSCC [9-11]. 
In humans, the Hh pathway functions in primary cilia, the organelles extending from the plasma membrane. Hh ligand binding to the Patched transmembrane receptor (PTCH1) leads to the ciliary accumulation of Smoothened (SMO) and its subsequent activation. SMO activation transduces the $\mathrm{Hh}$ signal to the cytoplasm, resulting in the nuclear translocation of GLI-Krüppel transcription factors (GLI1, 2, or 3), particularly GLI1, which thereby induces the expression of genes targeted by the Hh pathway [12].

The Hh pathway can be activated by the homologous proteins Sonic Hedgehog (SHH) and Indian Hedgehog ( $\mathrm{IHH}$ ), which are involved in cellular differentiation, migration, and proliferation [13], the recruitment of peripheral monocytes [14], and the Hh pathway activation in M2 macrophages [15]. This pathway has also been recently linked to angiogenesis $[16,17]$ and endothelial cell activation $[18,19]$. In tumors, M2 macrophages, also known as tumor-associated macrophages (TAM), exhibit pro-tumorigenic activity [20-24] that favors tumor progression [25] by way of a variety of mechanisms, including the stimulation of angiogenesis [26-28].

Considering that the Hh pathway participates in the pathogenesis of OSCC and that TAMs may mediate the abnormal activation of this signaling pathway and/or be responsive to it, the present study aimed to assess immunoexpression in the primary Hh pathway components (SHH, IHH, and GLI1) by investigating associations between these molecules and the clinical aspects of OSCC, angiogenesis, and CD163+ macrophages.

\section{Materials and methods}

The present research proposal received approval from our host institution's review board. Informed consent was obtained from all individual participants included in the study. The immunoexpression of proteins SHH, IHH, GLI1, CD163, and CD105 was studied in 28 cases of OSCC. All tumors were clinically classified by two experienced pathologists according to the TNM Classification of Malignant Tumours (2002), in addition to being histologically classified in accordance with WHO criteria (2005). After reviewing all 28 resection margins, tumor-free resection margins (TM) were seen in nine cases of OSCC, while five (55.55\%) of these presented oral epithelial dysplasia (OED). Three (60\%) of these OED cases were classified as mild, one (20\%) as moderate, and another $(20 \%)$ as severe. In addition, we compared the expression of the above proteins with four histologically normal nonneoplastic oral mucosa (NNM) tissue samples selected from the removal sites of unerupted mandibular third molars of ten healthy patients, i.e., non-smokers who refrained from alcohol use. Table 1 lists the clinical and histological characteristics of all 28 OSCC cases studied.
Table 1 Clinical and histological characteristics of patients with OSCC

\begin{tabular}{|c|c|c|}
\hline Clinical parameters & Total $(n)$ & Percent \\
\hline \multicolumn{3}{|l|}{ Gender } \\
\hline Males & 20 & 71.42 \\
\hline Females & 8 & 28.58 \\
\hline \multicolumn{3}{|l|}{ Size } \\
\hline $\mathrm{T} 1-\mathrm{T} 2$ & 14 & 50 \\
\hline $\mathrm{T} 3-\mathrm{T} 4$ & 14 & 50 \\
\hline \multicolumn{3}{|l|}{ Metastasis - lymph node } \\
\hline N0 & 7 & 35 \\
\hline N1-N3 & 21 & 75 \\
\hline \multicolumn{3}{|l|}{ Metastasis - distance } \\
\hline M0 & 23 & 82.14 \\
\hline Mx & 5 & 17.86 \\
\hline \multicolumn{3}{|l|}{ Clinical status } \\
\hline I-II & 3 & 10.72 \\
\hline III-IV & 25 & 89.28 \\
\hline \multicolumn{3}{|l|}{ Muscular invasion } \\
\hline Yes & 13 & 46.42 \\
\hline No & 15 & 53.58 \\
\hline \multicolumn{3}{|l|}{ Bone invasion } \\
\hline Yes & 4 & 14.28 \\
\hline No & 24 & 85.72 \\
\hline \multicolumn{3}{|c|}{ Perineural and vascular invasion } \\
\hline Yes & 2 & 7.14 \\
\hline No & 26 & 92.86 \\
\hline \multicolumn{3}{|l|}{ Histological gradation } \\
\hline Well differentiated & 19 & 67.85 \\
\hline Moderately differentiated & 8 & 28.58 \\
\hline Poorly differentiated & 1 & 3.57 \\
\hline \multicolumn{3}{|l|}{ Anatomic site } \\
\hline Tongue & 15 & 53.57 \\
\hline Floor of mouth & 8 & 28.57 \\
\hline Retromolar region & 3 & 10.71 \\
\hline Gingiva & 2 & 7.15 \\
\hline
\end{tabular}

\section{Immunohistochemistry}

Sections $4-\mu \mathrm{m}$ thick were obtained from formalin-fixed paraffin-embedded specimens. Histological sections were deparaffinized in xylene, then rehydrated with alcohol, and subjected to antigen retrieval (SHH, IHH, GLI1, CD163citrate buffer $\mathrm{pH}$ 6.0; CD105-citrate buffer $\mathrm{pH}$ 9.0) under moist heat for $45 \mathrm{~min}$. Endogenous peroxidase activity was subsequently blocked (Peroxidase Blocking Solution ${ }^{\mathrm{TM}}$, Dako, Carpinteria, USA) for $10 \mathrm{~min}$, and the primary antibodies were incubated overnight $(18 \mathrm{~h})$ at $4{ }^{\circ} \mathrm{C}(\mathrm{SHH}$, Novus Biologicals, Clone 5H4, 1:1000; IHH, Novus Biologicals, 
Clone EP1192Y, 1:500; GLI1, Novus Biologicals, Polyclonal, 1:600; CD105, Dako, Clone SN6h, 1:50; CD163, SantaCruz, Clone GH1/61, 1:50). Next, HRP Link and HRP Enzyme reagents (Advance ${ }^{\mathrm{TM}}$, Dako Corporation, Carpinteria, USA) were applied to tissue sections for $20 \mathrm{~min}$ each. Reactions were developed using 3,3-diaminobenzidine (Dako, Carpinteria, USA) and then counterstained with Harris hematoxylin. As a negative control, each primary antibody was substituted by normal serum of the same isotype.

\section{Double staining for $\mathrm{SHH} / \mathrm{CD} 163, \mathrm{IHH} / \mathrm{CD} 163$, GLI1/CD163, and GLI1/CD105}

To colocalize the proteins SHH/CD163, IHH/CD163, and GLI1/CD163 in OSCC samples, the EnVision ${ }^{\mathrm{TM}} \mathrm{G} \mid 2$ Doublestain and Dako Advance ${ }^{\mathrm{TM}}$ polymeric amplification systems (Dako, Carpinteria, USA) were used with Permanent Red and Vina Green chromogens in accordance with manufacturer instructions. GLI1/CD105 protein colocalization was identified using the Advance ${ }^{\mathrm{TM}}$ system (Dako, Carpinteria, USA) and MACH $3^{\text {TM }}$ Alkaline Phosphatase (Biocare Medical, Concord, CA, USA) with 3,3-diaminobenzidine and Ferangi Blue chromogens, in accordance with the manufacturer's instructions. All slides were mounted in $10 \%$ glycerol.

\section{Immunohistochemical analysis}

An Aperio digital microscope (Leica Microsystems, Wetzlar, Germany) was used to scan all slides, which were displayed on an LCD monitor via Aperio ImageScope software (Leica Microsystems, Wetzlar, Germany). To evaluate the SHH, IHH, and GLI1 proteins, five coincident and representative areas ( $\times 200$ final amplification) of each OSCC case were selected. These analyses were likewise performed for all TM and NNM cases:

(a) Analysis of SHH, IHH, and GLI1 proteins

The location of each protein was noted, whether in the membrane and/or cytoplasm (SHH and $\mathrm{IHH}$ ), or in the cytoplasm and/or nucleus (GLI1). In the parenchyma/epithelium, positive and negative cells were first counted in each microscopic field. Next, the number of positive cells was divided by the total number of cells, and semiquantification criteria, as previously described by Gurgel et al. [29], were applied to obtain scores as follows: negative $(-),<5 \%$ of positively immunostained cells; $1+, 5$ $25 \%$; 2+, 26-50\%; 3+, 51-75\%; and 4+, >75\% of positively immunostained cells. In the stroma/lamina propria, endothelial cells expressing $\mathrm{SHH}, \mathrm{IHH}$, and GLI1 were counted in the previously selected densely vascular CD105 protein foci (i.e., "hot-spot" areas).

(b) CD163 and CD105 protein analysis

Five areas of tumor stroma with the highest populations of CD163+ cells were selected. These same sites were then analyzed at a final amplification of $\times 200$ by counting CD163+ cells and CD105+ vessels. Any blood vessels that were illuminated and brownish in color were considered positive. These areas were subsequently measured, and final results were expressed as CD163+ infiltrating macrophage density (IMD) per square millimeter and microvascular density (MVD) per square millimeter.

\section{Statistical analysis}

All data were submitted to statistical analysis under nonparametric testing (Mann-Whitney, Kruskal-Wallis, and Spearman correlation) using GraphPad Prism Software (GraphPad Software, San Diego, USA) and $p$ values corresponding to alpha $(\alpha)$, i.e., less than or equal to $5 \%$, were considered significant.

\section{Results}

\section{Hh pathway reactivation in OSCC}

\section{SHH}

In tumor parenchyma, the $\mathrm{SHH}$ protein was observed in the cytoplasm of neoplastic cells with positive scores in $25 \mathrm{OSCC}$ cases $(89.28 \%)$, with $4+$ being the predominantly assigned score $(n=17 ; 68 \%)$, followed by $3+(n=5 ; 20 \%), 1+(n=2$; $8 \%)$, and $2+(n=1,4 \%)$. In TM, six cases $(66.66 \%)$ demonstrated cytoplasmic immunostaining for the SHH protein in the epithelial compartment, with predominant scores of $2+$ $(n=2 ; 33.33 \%)$ and $3+(n=2 ; 33.33 \%)$, followed by $1+$ $(n=1,16.67 \%)$ and $4+(n=1,16.67 \%)$. All NNM cases were found to be negative for this protein (Fig. 1).

\section{$I H H$}

Twenty-two OSCC cases (78.57\%) received positive scores with respect to the IHH protein in tumor parenchyma, with 4+ $(n=7 ; 31.82 \%)$ and $3+(n=7 ; 31.82 \%)$ being the predominant scores, followed by $1+(n=5 ; 22.72 \%)$ and $2+(n=3$; $13.64 \%$ ). Although IHH was generally seen in the cytoplasm, it was also present in the cellular membrane in three $(13.64 \%)$ cases. Eight TMs $(88.88 \%$ ) demonstrated cytoplasmic immunostaining for IHH in the epithelial compartment, with a score of $1+(n=4,50 \%)$ predominating, followed by scores of $2+$ $(n=2 ; 25 \%)$ and $3+(n=2 ; 25 \%)$. Three $(75 \%)$ cases of NNM exhibited cytoplasmic labeling exclusively in the epithelium, scored as 1+ (Fig. 1). 
NNM
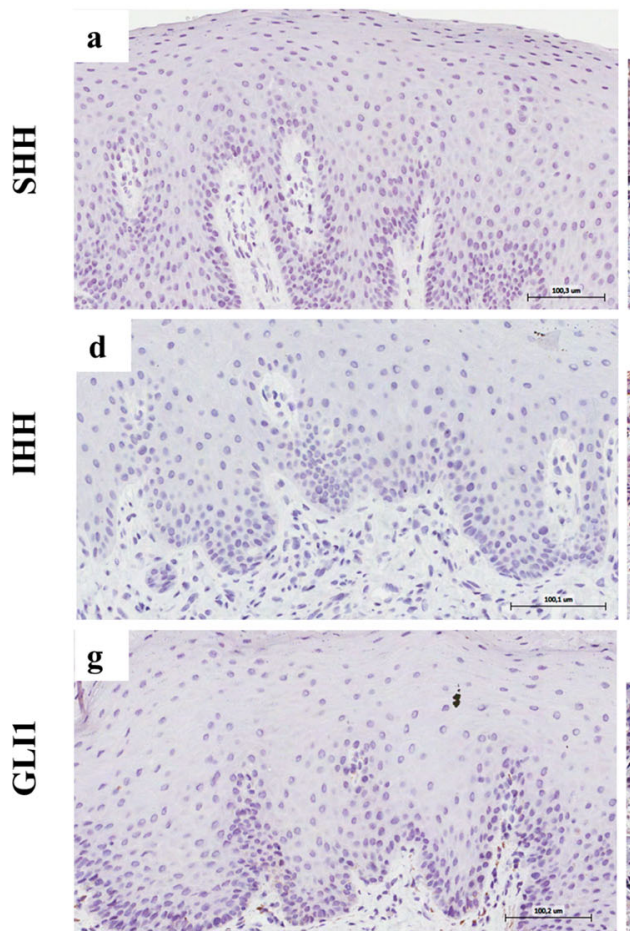

Fig. 1 Immunostaining for SHH, IHH, and GLI1 in cases of NNM, TM, and OSCC. SHH: a negatively scored non-neoplastic oral mucosa (NNM). b, c Tumor margin (TM) and oral squamous cell carcinoma (OSCC) samples exhibiting cytoplasmic staining scored $2+$ and 4+, respectively. IHH: d epithelial cells scored negatively in NNM cases. e,

\section{GLII}

The GLI1 protein was positively scored in tumor parenchyma in $24(85.72 \%)$ of the OSCC cases, with a predominant score of $4+(n=15 ; 62.5 \%)$, followed by $3+(n=7 ; 29.16 \%), 1+$ $(n=1,4.17 \%)$, and $2+(4.17 \%)$. This protein was found to be present in both the nuclei and cytoplasm of tumor cells. In the epithelium, TMs showed positivity in seven $(77.77 \%)$ cases, with scores of $1+(n=3 ; 42.86 \%), 2+(n=2 ; 28.57 \%)$, and $4+$ $(n=2 ; 28.57 \%)$. By contrast, just one $(25 \%)$ of the NNM cases exhibited cytoplasmic and nuclear staining exclusively in the epithelium, with a score of 1+ (Fig. 1).

Figure 2 shows comparisons among the Hedgehog molecules in OSCC, TM, and NNM. Tumors showed higher expression of SHH ( $p=0.016)$ and GLI1 $(p=0.03)$, than TM and NNM. OSCC also have a higher number of IHH positive cells, but it was not significant in comparison to TM and NNM (Fig. 2; $p=0.39$ ).

\section{Hh molecules are present in newly formed vessels within OSCC stroma}

Endothelial cells expressing SHH, IHH, and GLI1 were seen within CD105+ vessels (Fig. 3), with correlations observed between the proteins CD105 and GLI1 $(r=0.54 ; p=0.002)$
TM

OSCC
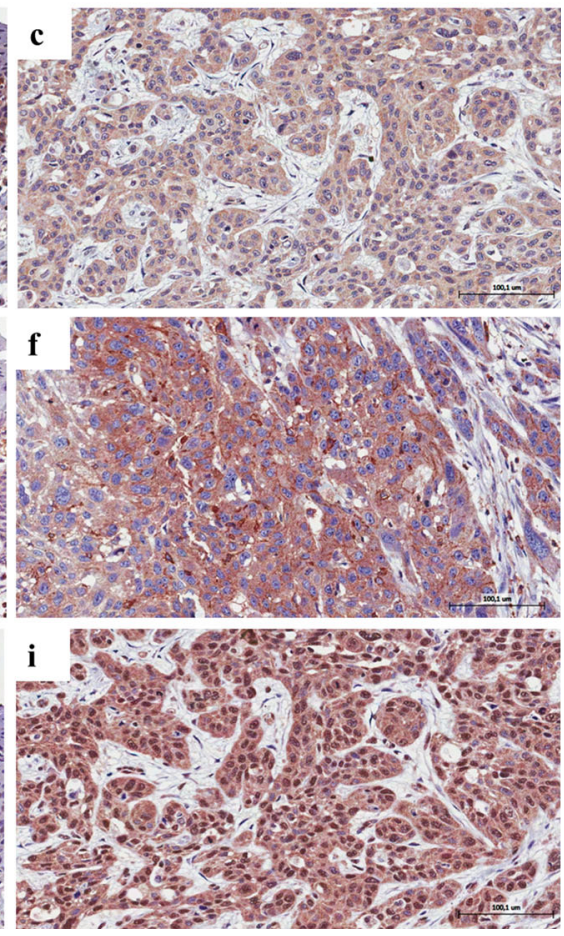

f Cytoplasmic immunostaining in TM and OSCC with scores of 1+ and 4+, respectively. GL11: g negatively scored NNM. h, i Nuclear and cytoplasmic immunostaining in TM and OSCC with scores of $1+$ and $4+$, respectively

and CD105 and IHH $(r=0.39 ; p=0.03)$. Likewise, Hedgehog molecules were also found to be present in $\mathrm{CD} 105+$ vessels in TMs, albeit to a lesser extent ( $n=4 ; 44.44 \%)$.

Within OSCC stroma, immunostaining for the protein GLI1, a Hh pathway transcription factor, was primarily observed in the nuclei of endothelial cells. Protein colocalization demonstrated the presence of GLI1 in the nuclei of endothelial cells, while CD105 was observed in the cytoplasm of these same cells. Figure $4 a-d$ depicts positively stained CD105, $\mathrm{SHH}, \mathrm{IHH}$, and GLI1 proteins in blood vessels located within

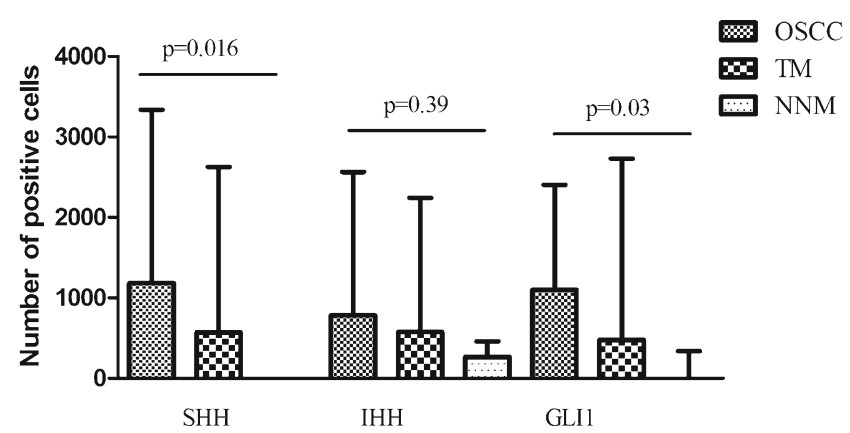

Fig. 2 Distribution of Hedgehog protein expression in cases of OSCC, TM, and NNM. Median (range) values of the number of positive cells are shown. Note: The median of immunostaining for the SHH and GLI1 proteins in NNM were zero 


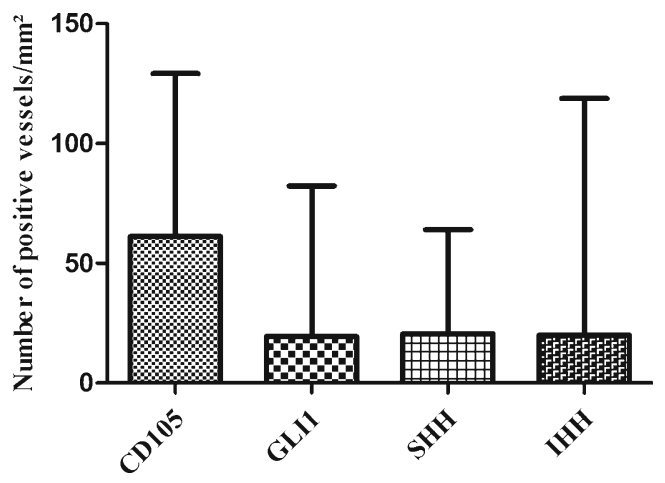

Fig. 3 Number of vessels expressing CD105, GLI1, SHH, and IHH in the stroma of OSCC. Median (range) values are shown

tumor stroma, as well as the colocalization of GLI1 and CD105 in endothelial cells (e-f).

\section{CD163+ macrophages express the IHH ligand and influence angiogenesis in OSCC}

The present investigation found an association between CD105+ vessels and the presence of CD163+ macrophages in the stroma adjacent to tumor islands in OSCC. A correlation was observed between IMD and MVD in OSCC cases $(r=0.77 ; p<0.0001)$ (Figs. 5 and 6$)$, as well as in TMs $(r=1.0 ; p<0.0001)$.

Fig. 4 Immunoexpression of CD105, SHH, IHH, and GLI1 proteins in blood vessels within OSCC stroma. a-d Cytoplasmic immunostaining in endothelial cells adjacent to tumor islands. e, $\mathbf{f}$ Double staining indicating the presence of CD105 (Ferangi Blue) in the cytoplasm and GLI1 (DAB) in the nucleus of an endothelial cell

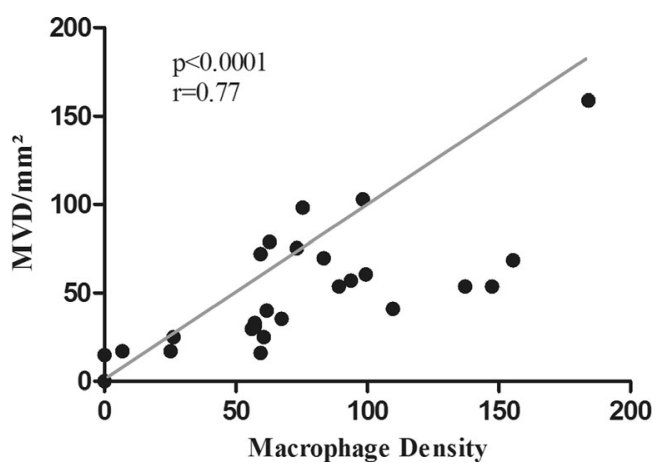

Fig. 5 Correlation between infiltrating macrophage density and microvascular density in OSCC

Protein colocalization analysis was employed to assess whether macrophages are capable of producing Hh pathway components (SHH, IHH, and GLI1). Evaluation of SHH/ CD163, IHH/CD163, and GLI1/CD163 indicated the exclusive presence of the IHH ligand in CD163+ macrophages. By simultaneously immunostaining for IHH and CD163 in a given cell, immunohistochemical labeling is evidenced as a result of the particular chromogen mixture used (Fig. 6). Individual staining for IHH (Vina Green) and CD163 (Red Permanent) can be seen in the supplemental Figure 1.

Supplemental Table 1 illustrates the expression of SHH, IHH, GLI1, CD163 (IMD), and CD105 (MVD) in accordance
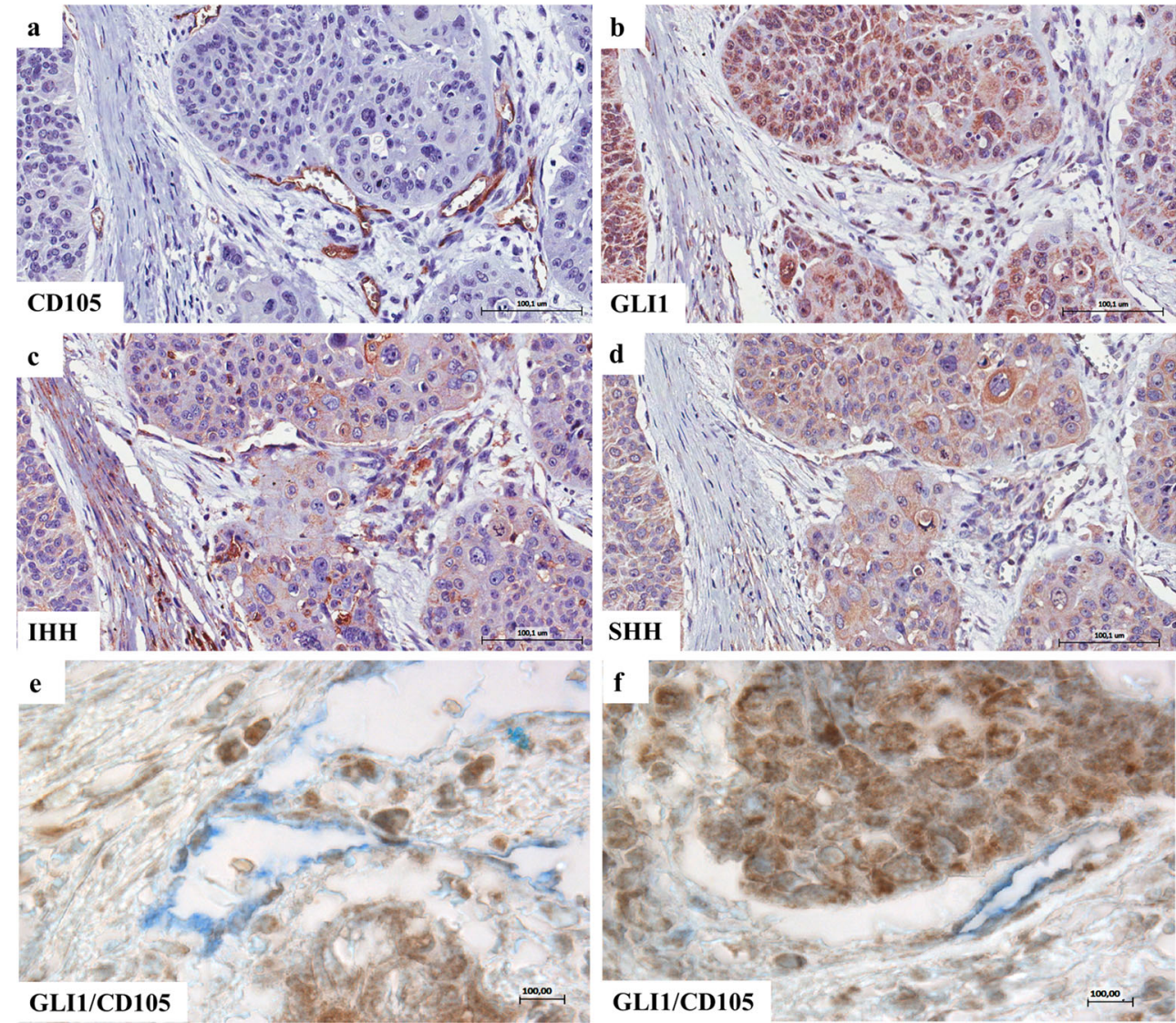
Fig. 6 CD105 and CD163 protein expression in blood vessels within OSCC stroma. a, c Cytoplasmic immunostaining in endothelial cells adjacent to tumor islands. b, d CD163 immunoreactivity evident in the membrane and cytoplasm in OSCC cases. e-h Double staining indicating the presence of CD163 (Permanent Red) and IHH (Vina Green) in the cytoplasm of OSCC stroma cells
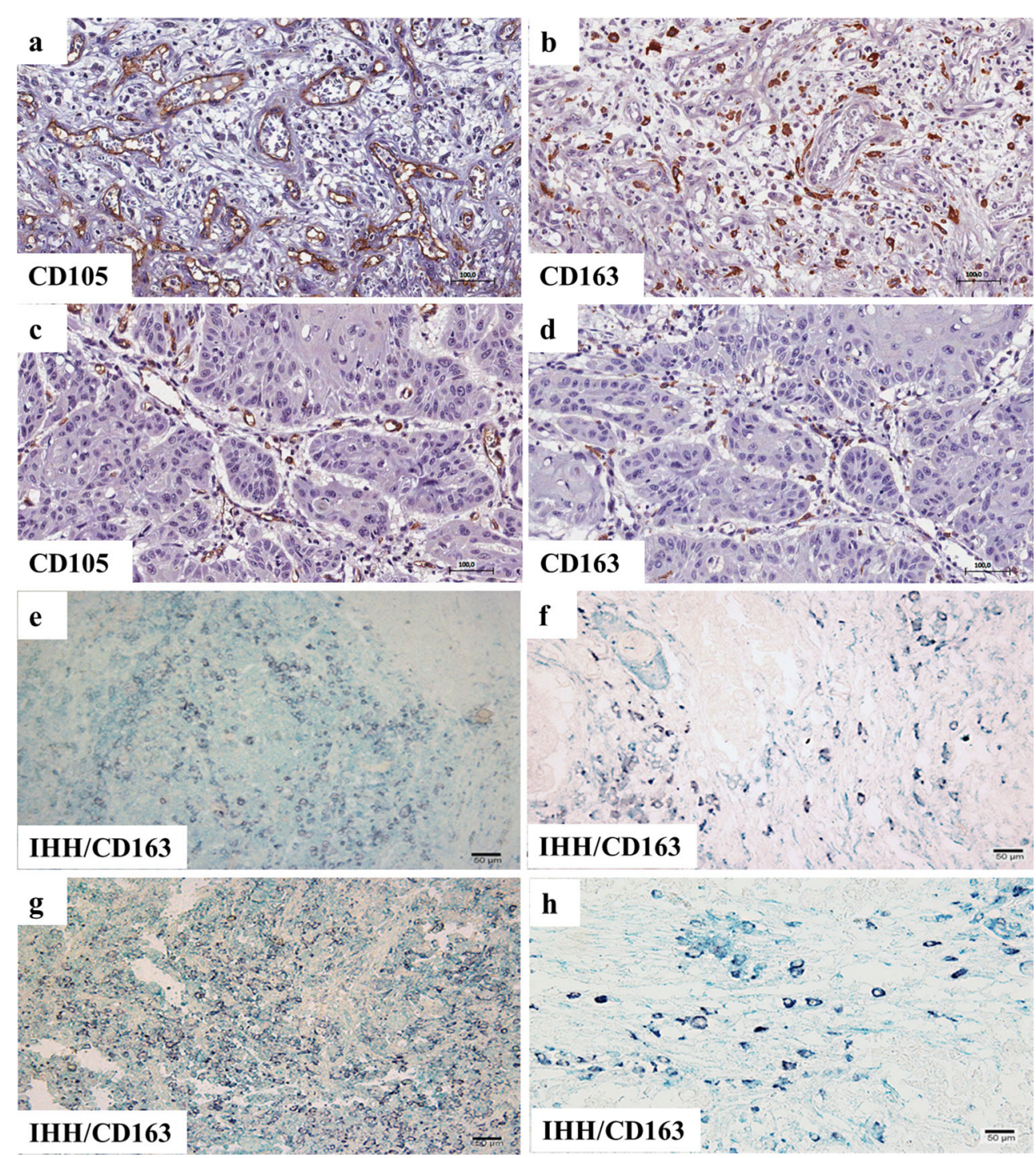

with the clinical and histological parameters in OSCC cases and supplemental Table 2 shows a comparison of the expression of all these proteins in OSCC, TM, and MNN.

\section{Discussion}

The present results corroborate recently published findings indicating the reactivation of the Hh pathway in OSCC, which appears to participate in the pathogenesis of this disease [9-11, 30]. Furthermore, the proteins involved in this pathway (SHH, IHH, and GLI1) were found to be overexpressed in OSCC tumors in comparison to TMs and NNMs. Additionally, the observation of Hh pathway components in endothelial cells leads us to believe that these molecules are capable of contributing to tumor angiogenesis, since CD163+ macrophages express $\mathrm{IHH}$, a pathway ligand, and are known to be associated with tumor neovascularization [21, 22, 24].

In normal adult tissue, the signaling pathways that participate in embryonic development, such as the Hh pathway, normally remain inactive except when participating in tissue repair or stem cell maintenance [31-33]. When deregulated, a variety of mechanisms are capable of activating the Hh pathway, some of which are dependent on the Hh ligand. Liganddependent mechanisms may prove to be more complex in nature, since these involve not only the tumor itself but also the tumoral microenvironment and possibly even other signaling pathways [33].

The immunoexpression of SHH and IHH proteins observed in the cytoplasm of neoplastic cells herein is suggestive of Hh pathway reactivation in OSCC, since the ligands are internalized upon interaction with the PTCH1 receptor. Additionally, the presence of these morphogens, most notably $\mathrm{IHH}$, 
in OSCC tumor parenchyma and stroma indicates the potential occurrence of autocrine and paracrine pathway activation, similarly to what is seen in hepatocellular carcinoma [34], pancreatic cancer [35], and breast cancer [36]. Thus, it is plausible that the role of the Hh pathway may extend beyond the parenchyma, possibly involving the entire tumor microenvironment. Moreover, Pereira et al. [37] likewise reported greater IHH immunoreactivity in stromal cells when compared to $\mathrm{SHH}$.

The finding of nuclear immunostaining for GLI1 serves to confirm the participation of the Hh pathway in OSCC, as the translocation of this protein occurs only following the activation of this pathway's signaling cascade $[12,38]$. In addition to the nucleus, GLI1 was also observed in the cytoplasm. In this cellular compartment, the absence of SHH or IHH ligands provokes GLI proteins to undergo proteolytic processing, resulting in a loss of activation domain, which then causes them to act as transcriptional repressors [39]. Regardless, it is worth noting that the GLI transcription factors (GLI1, GLI2, and GLI3) are encoded by distinctly different genes [12, 33]. Since GLI1 does not possess an N-terminal repressor domain, it functions exclusively as an activator of this pathway [31]. Accordingly, the presence of GLI1 in the cytoplasm also appears to suggest the need for cytoplasmic accumulation prior to nuclear translocation, as similarly observed with respect to other transcription factors, such as p53 [40].

Despite the comparatively high levels of Hh pathway protein expression seen in the OSCC cases considered herein, most of the TMs also exhibited immunostaining for $\mathrm{SHH}$ $(n=6 ; 66.66 \%)$, IHH $(n=8 ; 88.88 \%)$, and GLI1 $(n=7$; $77.77 \%$ ). This finding appears to corroborate the theory espoused by Leovic et al. [11] known as "field cancerization," in which distinct areas of head and neck squamous cell carcinoma become exposed to carcinogens for extended periods, resulting in genetically altered fields capable of developing carcinomas. The present results would seem to indicate that Hh pathway activation also occurs in TMs, thereby suggesting the presence of genetic alterations in cells proximate the tumor site, despite the apparent absence of any cytological and/or architectural alterations. Of the nine TM cases studied, five had oral epithelial dysplasia concomitant with elevated SHH, IHH, and GLI1 protein expression. Furthermore, Dias et al. [30] recently demonstrated that the Hh pathway can be activated in OED, a stage precedent to carcinoma development.

The GLI1 protein was identified in the nuclei of endothelial cells within tumor stroma, while the SHH and IHH ligands were observed in the cytoplasm of these same cells. According to Harris et al. [41], endothelial cells do not appear to naturally express or produce $\mathrm{Hh}$ molecules. However, in the context of chronic viral hepatitis, the expression of GLI2 transcription factor in endothelial cells has been positively demonstrated [37]. Furthermore, Olsen et al. [18] identified endothelial cells in tumor stroma presenting elevated levels of
PTCH1 expression and low levels of HHIP, a Hh pathway inhibitor.

These findings, when taken together, would seem to indicate that, similarly to the role played in blood vessel formation in embryonic tissue (vasculogenesis), the Hh pathway appears to become reactivated in order to participate in tumor neovascularization. The present results provide evidence of the activation of this signaling cascade in endothelial cells within the tumor stroma of OSCC, since, in addition to the cytoplasmic immunostaining observed for the pathway ligands, GLI1 transcription factor was also identified in the nuclei of these cells. Moreover, the colocalization of GLI1 and CD105 proteins in endothelial cells serves to additionally confirm the role played by the Hh pathway in tumoral angiogenesis.

Previous studies have demonstrated that TAMs are mostly polarized to the $\mathrm{M} 2$ phenotype and thusly present pro-tumoral functions [20-24]. In comparison to TMs and NNMs, the OSCC cases exhibited greater CD163+ macrophage density, suggesting that these cells may potentially play a role in oral carcinogenesis. Few studies have evaluated IMD in OSCC [42-46], and no studies to date have attempted to investigate whether these cells are responsive to and/or secrete Hh pathway proteins in these types of tumors.

Among the findings presented herein, the fact that CD163+ macrophages express IHH suggests that Hh signaling plays a role not only in neoplastic cell growth but also in tumoral microenvironment [47]. Several studies have recently identified Hh pathway receptors and intracellular components in macrophages, indicating that these cells are capable of transducing signaling initiated by Hh morphogens [14, 48]. Pereira et al. [15] also demonstrated that, in human schistosomiasis, macrophages produce IHH, which in turn promotes M2 profile activation, fibrogenesis, and angiogenesis.

It has been well established that cells involved in innate immune response, e.g., macrophages, play a role in mediating the angiogenic "switch," thereby favoring tumor progression. Moreover, these cells can also assist in local invasion and hinder the action of drugs targeting the inhibition of endothelial activation [25, 49]. Jurisic et al. [50], for example, found that in radicular cysts, the concentration of TNF- $\alpha$ correlate with number inflammatory cells (macrophages) and degree of vascularization. Our present investigation found a higher density of vessels per square millimeter in OSCCs compared to TMs and NNMs, substantiating the role played by angiogenesis in promoting an environment suitable for tumor growth. Moreover, MVD has also been associated with the presence of CD163+ macrophages in the stroma adjacent to tumor islands, indicating that macrophages do, in fact, appear to contribute to the modulation of the microenvironment in oral carcinogenesis, which also consequently favors tumor progression. Although commonly associated with solid tumor growth, recent studies have reported that the formation of blood vessels prior to macroscopic identification of malignancy also seems to be a 
contributing factor in the transformation and growth of neoplastic cells [25]. The results reported herein corroborate this postulation, since an association was also observed between MVD and IMD in the lamina propria of TMs.

In sum, the present investigation serves to contribute to the body of knowledge surrounding the participation of the $\mathrm{Hh}$ pathway in OSCC by providing evidence supporting the reactivation of this pathway via autocrine and paracrine signaling. We further demonstrate that the Hh pathway is also expressed in endothelial cells, which may contribute to angiogenesis. In addition, CD163+ macrophages not only were observed to express IHH, a ligand of this pathway, but also were found to be associated with tumor neovascularization. Considering this, many components of the tumoral microenvironment have been shown to be crucial to the process of carcinogenesis and, as such, represent additional therapeutic targets [51]. With respect to macrophages, for example, the inhibition of M2 polarization and pro-tumoral activity, as well as targeting increased expression of the M1 phenotype concurrent with cytotoxic functions, both represent promising strategies in the treatment of human tumors [21, 52]. Furthermore, the identification of GLI1 in the nuclei of endothelial cells suggests that Hh pathway inhibition may prove useful in several types of neoplasia dependent on angiogenesis for progression [18]. Therefore, tumor growth may be suppressed by blocking the Hh pathway, both directly, by interfering with intratumoral survival, and indirectly, by altering the tumoral microenvironment.

Acknowledgments This study was supported by grants from Foundation for Search of Bahia State-FAPESB, Bahia, Brazil (007/2013), and National Council for Scientific and Technological Development, CNPq, Brazil (446065/2014-5). The authors would like to thank Andris K. Walter for providing English translation and consulting services.

\section{Compliance with ethical standards}

\section{Conflicts of interest None}

Ethics approval and consent to participate The present research proposal received approval from our host institution's review board. Informed consent was obtained from all individual participants included in the study.

\section{References}

1. Bhargava A, Saigal S, Chalishazar M. Histopathological grading systems in oral squamous cell carcinoma: a review. J Int Oral Health. 2010;2:1-10.

2. Jemal A, Bray F, Center MM, Ferlay J, Ward E, Forman D. Global cancer statistics. CA Cancer J Clin. 2011;61(2):69-90.

3. Adrien J, Bertolus C, Gambotti L, Mallet A, Baujat B. Why are head and neck squamous cell carcinoma diagnosed so late?
Influence of health care disparitiesand socio-economic factors. Oral Oncol. 2014;50:90-7.

4. Rodrigues PC, Miguel MC, Bagordakis E, Fonseca FP, de Aquino $\mathrm{SN}$, Santos-Silva AR, et al. Clinicopathological prognostic factors of oral tongue squamous cell carcinoma: a retrospective study of 202 cases. Int J Oral Maxillofac Surg. 2014;43:795-801.

5. Ingham PW, McMahon AP. Hedgehog signaling in animal development: paradigms and principles. Genes Dev. 2001;15:3059-87.

6. Thayer SP, di Magliano MP, Heiser PW, Nielsen CM, Roberts DJ, Lauwers GY, et al. Hedgehog is an early and late mediator of pancreatic cancer tumorigenesis. Nature. 2003;425:851-6.

7. Chari NS, McDonnell TJ. The sonic hedgehog signaling network in development and neoplasia. Adv Anat Pathol. 2007;14:344-52.

8. Hwang J, Kang MH, Yoo YA, Quan YH, Kim HK, Oh SC, et al. The effects of sonic hedgehog signaling pathway components on non-small-cell lung cancer progression and clinical outcome. World J Surg Oncol. 2014;12:268.

9. Cavicchioli Buim ME, Gurgel CAS, Gonçalves Ramos EA, Lourenço SV, Soares FA. Activation of sonic hedgehog signaling in oral squamous cell carcinomas: a preliminary study. Hum Pathol. 2011;42:1484-90.

10. Honami T, Shimo T, Okui T, Kurio N, Hassan NM, Iwamoto M, et al. Sonic hedgehog signaling promotes growth of oral squamous cell carcinoma cells associated with bone destruction. Oral Oncol. 2012;48:49-55.

11. Leovic D, Sabol M, Ozretic P, Musani V, Car D, Marjanovic K, et al. Hh-Gli signaling pathway activity in oral and oropharyngeal squamous cell carcinoma. Head Neck. 2012;34:104-12.

12. Hassounah NB, Bunch TA, McDermott KM. Molecular pathways: the role of primary cilia in cancer progression and therapeutics with a focus on Hedgehog signaling. Clin Cancer Res. 2012;18:242935 .

13. Van den Brink GR, Bleuming SA, Hardwick JC, Schepman BL, Offerhaus GJ, Keller JJ, et al. Indian Hedgehog is an antagonist of Wnt signaling in colonic epithelial cell differentiation. Nat Genet. 2004;36:277-82.

14. Dunaeva M, Voo S, van Oosterhoud C, Waltenberguer J. Sonic hedgehog is a potente chemoattractant for human monocytes: diabetes mellitus inhibits Sonic hedgehog-induced monocyte chemotaxis. Basic Res Cardiol. 2010;105:61-71.

15. Pereira TA, Xie G, Choi SS, Syn WK, Voieta I, Lu J, et al. Macrophage-derived Hedgehog ligands promotes fibrogenic and angiogenic responses in human schistosomiasis mansoni. Liver Int. 2013;33(1):149-61.

16. Pinter M, Sieghart W, Schmid M, Dauser B, Prager G, Dienes HP, et al. Hedgehog inhibition reduces angiogenesis by downregulation of tumoral VEGF-A expression in hepatocellular carcinoma. United European Gastroenterol J. 2013;1:265-75.

17. Mathew E, Zhang Y, Holtz AM, Kane KT, Song JY, Allen BL, et al. Dosage-dependent regulation of pancreatic cancer growth and angiogenesis by hedgehog signaling. Cell Rep. 2014;9:484-94.

18. Olsen CL, Hsu PP, Glienke J, Rubanyi GM, Brooks AR. Hedgehog-interacting protein is highly expressed in endothelial cells but down-regulated during angiogenesis and in several human tumors. BMC Cancer. 2004;4:43.

19. Vokes SA, Yatskievych TA, Heimark RL, McMahon J, McMahon AP, Antin PB, et al. Hedgehog signaling is essential for endothelial tube formation during vasculogenesis. Development. 2004;131: 4371-80.

20. Marioni G, D’Alessandro E, Giacomelli L, Staffieri A. CD105 is a marker of tumour vasculature and a potential target for the treatment of head and neck squamous cell carcinoma. J Oral Pathol Med. 2010;39:361-7.

21. Shirabe K, Mano Y, Muto J, Matono R, Motomura T, Toshima T, et al. Role of tumor-associated macrophages in the progression of hepatocellular carcinoma. Surg Today. 2012;42:1-7. 
22. Obeid E, Nanda R, Fu YX, Olopade OI. The role of tumorassociated macrophages in breast cancer progression (review). Int J Oncol. 2013;43:5-12.

23. He KF, Zhang L, Huang CF, Ma SR, Wang YF, Wang WM, et al. CD163+ tumor-associated macrophages correlated with poor prognosis and cancer stem cells in oral squamous cell carcinoma. Biomed Res Int. 2014;2014:838632.

24. Pedersen MB, Danielsen AV, Hamilton-Dutoit SJ, Bendix K, Nørgaard P, Møller MB, et al. High intratumoral macrophage content is an adverse prognostic feature in anaplastic large cell lymphoma. Histopathology. 2014;65:490-500.

25. Hanahan D, Weinberg RA. Hallmarks of cancer: the next generation. Cell. 2011;144:646-74.

26. Bingle L, Brown NJ, Lewis CE. The role of tumour-associated macrophages in tumour progression: implications for new anticancer therapies. J Pathol. 2002;196:254-65.

27. Guo C, Buranych A, Sarkar D, Fisher PB, Wang XY. The role of tumor-associated macrophages in tumor vascularization. Vasc Cell. 2013;5:20

28. Wang X, Zhao X, Wang K, Wu L, Duan T. Interaction of monocytes/macrophages with ovarian cancer cells promotes angiogenesis in vitro. Cancer Sci. 2013;104:516-23.

29. Gurgel CA, Ramos EA, Azevedo RA, Sarmento VA, da Silva Carvalho AM, dos Santos JN. Expression of Ki-67, p53 and p63 proteins in keratocyst odontogenic tumours: an immunohistochemical study. J Mol Histol. 2008;39:311-6.

30. Dias RB, Valverde LF, Sales CBS, Guimarães VS, Cabral MG, de Aquino Xavier FC, et al. Enhanced expression of hedgehog pathway proteins in oral epithelial dysplasia. Appl Immunohistochem Mol Morphol. 2015

31. Cohen DJ. Targeting the hedgehog pathway: role in cancer and clinical implications of its inhibition. Hematol Oncol Clin North Am. 2012;26:565-88.

32. McMillan R, Matsui W. Molecular pathways: the hedgehog signaling pathway in cancer. Clin Cancer Res. 2012;18:4883-8.

33. Amakye D, Jagani Z, Dorsch M. Unraveling the therapeutic potential of the Hedgehog pathway in cancer. Nat Med. 2013;19:1410 22.

34. Chan IS, Guy CD, Chen Y, Lu J, Swiderska-Syn M, Michelotti GA, et al. Paracrine Hedgehog signaling drives metabolic changes in hepatocellular carcinoma. Cancer Res. 2012;72:6344-50.

35. Sahebjam S, Siu LL, Razak AA. The utility of hedgehog signaling pathway inhibition for cancer. Oncologist. 2012;17:1090-9.

36. O'Toole AS, Machalek DA, Shearer RF, Millar EK, Nair R, Schofield $\mathrm{P}$, et al. Hedgehog overexpression is associated with stromal interactions and predicts for poor outcome in breast cancer. Cancer Res. 2011;71:4002-14.

37. Pereira TA, Witek RP, Syn WK, Choi SS, Bradrick S, Karaca GF, et al. Viral factors induce Hedgehog pathway activation in humans with viral hepatitis, cirrhosis, and hepatocellular carcinoma. Lab Investig. 2010;90:1690-703.
38. Robbins DJ, Fei DL, Riobo NA. The Hedgehog signal transduction network. Sci Signal. 2012;5(246):re6.

39. Sheng T, Chi S, Zhang X, Xie J. Regulation of Gli1 localization by the cAMP/protein kinase A signaling axis through a site near the nuclear localization signal. J Biol Chem. 2006;281:9-12.

40. Gravina GL, Senapedis W, McCauley D, Baloglu E, Shacham S, Festuccia C. Nucleo-cytoplasmic transport as a therapeutic target of cancer. J Hematol Oncol. 2014;7:85.

41. Harris LG, Pannell LK, Singh S, Samant RS, Shevde LA. Increased vascularity and spontaneous metastasis of breast cancer by hedgehog signaling mediated upregulation of cyr61. Oncogene. 2012;31: 3370-80.

42. Mori K, Hiroi M, Shimada J, Ohmori Y. Infiltration of $\mathrm{m} 2$ tumorassociated macrophages in oral squamous cell carcinoma correlates with tumor malignancy. Cancers (Basel). 2011;3:3726-39.

43. Fujii N, Shomori K, Shiomi T, Nakabayashi M, Takeda C, Ryoke $\mathrm{K}$, et al. Cancer-associated fibroblasts and CD163-positive macrophages in oral squamous cell carcinoma: their clinicopathological and prognostic significance. J Oral Pathol Med. 2012;41:444-51.

44. França CM, Batista AC, Borra RC, Ventiades-Flores JA, Mendonça EF, Deana AM, et al. Macrophage migration inhibitory factor and oral cancer. J Oral Pathol Med. 2013;42:368-73.

45. Costa NL, Valadares MC, Souza PP, Mendonça EF, Oliveira JC, Silva TA, et al. Tumor-associated macrophages and the profile of inflammatory cytokines in oral squamous cell carcinoma. Oral Oncol. 2013;49:216-23.

46. Wang S, Sun M, Gu C, Wang X, Chen D, Zhao E, et al. Expression of CD163, interleukin-10, and interferon-gamma in oral squamous cell carcinoma: mutual relationships and prognostic implications. Eur J Oral Sci. 2014;122:202-9.

47. Chen W, Tang T, Eastham-Anderson J, Dunlap D, Alicke B, Nannini $\mathrm{M}$, et al. Canonical hedgehog signaling augments tumor angiogenesis by induction of VEGF-A in stromal perivascular cells. Proc Natl Acad Sci U S A. 2011;108:9589-94.

48. Schumacher MA, Donnelly JM, Engevik AC, Xiao C, Yang L, Kenny S, et al. Gastric Sonic Hedgehog acts as a macrophage chemoattractant during the immune response to Helicobacter pylori. Gastroenterology. 2012;142:1150-9.

49. Qian BZ, Pollard JW. Macrophage diversity enhances tumor progression and metastasis. Cell. 2010;141:39-51.

50. Jurisic V, Terzic T, Colic S, Jurisic M. The concentration of TNFalpha correlate with number of inflammatory cells and degree of vascularization in radicular cysts. Oral Dis. 2008;14(7):600-5.

51. Heller E, Hurchla MA, Xiang J, Su X, Chen S, Schneider J, et al. Hedgehog signaling inhibition blocks growth of resistant tumors through effects on tumor microenvironment. Cancer Res. 2012;72:897-907.

52. Merry R, Belfield L, McArdle P, McLennan A, Crean S, Foey A. Oral health and pathology: a macrophage account. Br J Oral Maxillofac Surg. 2012;50:2-7. 